\title{
Sleep Apnea-A Review
}

\author{
Rajbhandari $\mathbf{R}^{*}$ \\ *Shahid Gangalal National heart Centre
}

Corresponding: Dr. Rajeeb Rajbhandarii

Shahid Gangalal National Heart Centre, Bansbari, Kathmandu, Nepal Tel: 00977-1-4371322, 0097-1-4371374.

Fax: 00977-1-4371123

\section{INTRODUCTION}

Sleep apnea literally means "cessation of breath." Sleep apnea is a common disorder in which we have one or more pauses in breathing or shallow breaths during our sleep. Breathing pauses can last from a few seconds to minutes. They often occur 5 to 30 times or more an hour. Typically, normal breathing then starts again, sometimes with a loud snort or choking sound. Sleep apnea usually is a chronic condition that disrupts sleep 3 or more nights each week. This results in poor sleep quality that makes one tired during the day. Sleep apnea is one of the leading causes of excessive daytime sleepiness. The airway can become obstructed at several possible sites like the excess tissue in the airway enlarged tonsils, a large tongue, the airway muscle which relaxes and collapses during sleep.

The kind of repetitive episodes of upper airway obstruction that occur during sleep is usually associated with a reduction in blood oxygen saturation.

\section{OVERVIEW}

Sleep apnea often goes undiagnosed. It cant usually be detected during routine OPD visits. Also, there are no blood tests for the condition. Most people who have sleep apnea don't know they have it because it only occurs during sleep. A family member and/or bed partner usually first notice the signs of sleep apnea.. Obstructive sleep apnea happens more often in people who are overweight, but it can affect anyone.

Central sleep apnea is a less common type of sleep apnea. It happens when brainstem doesn't send the correct signals to your breathing muscles. Central sleep apnea often occurs with obstructive sleep apnea, but it can occur alone. Snoring doesn't typically happen with central sleep apnea.

This article mainly focuses on obstructive sleep apnea.

\section{Clinical features}

Common symptoms are excessive daytime sleepiness and frequent episodes of obstructed breathings during sleep that usually cause snoring.

Other features may include loud snoring, morning headaches, unrefreshing sleep, a dry mouth upon awakening, high blood pressure, overweight, irritability, depression, difficulty in concentrating, excessive perspiring during sleep, heartbutn, reduced libido, insomnia, nocturia, nocturnal snorting, gasping, choking, rapid weight gain etc.

\section{How serious is sleep apnea?}

It is a potentially life-threatening condition that requires immediate medical attention. The risk of undiagnosed obstructive sleep apnea include myocardial infarction, strokes, impotence, atrial fibrillation hypertension. In addition, obstructive sleep apnea causes daytime sleepiness that can result in accidents, lost productivity and interpersonal relationship problems. The severity of the symptoms may be mild, moderate of severe.

\section{Prognosis}

This type of nighttime breathing disorder usually manfestng as snoring increases a person's risk of having a myocardial infarction or sudden cardiac rrest by $30 \%$ over a period of four to five years, according to a new study.

Dr. Somers and other researchers think sleep apnea may increase some forms of heart disease because of hypoxia that occurs during sleep apnea. When oxygen level drops and carbon dioxide level increases, brain stem stimulates to release adrenaline-which increases blood pressure. Hypoxia also induces endothelial dysfunction that can eventually damage cardiovascular system and cause many heart problems.

\section{High blood pressure:}

Sudden drop in blood-oxygen levels that occur during sleep apnea increases blood pressure and strain the cardiovascular system. About half the people with sleep apnea develop hypertension.

\section{Heart failure:}

Sleep apnea may increase the risk of heart failure because of the swings in blood pressure that occur during sleep apnea; this, combined with hypoxia to myocardial tissue, might further damage heart muscles.

Atrial fibrillation is also common with this condition

\section{Coronary artery disease:}

Again, sleep apnea may increase the risk of coronary artery disease because of the swings in blood pressure that occur during sleep apnea. This in combination with dips in oxygen levels, might make it more susceptible to ischemia.

\section{Stroke:}

It is thought that hypertension blood pressure and hypoxia from sleep apnea, might make one more prone to stroke.

Untreated sleep spnea can also increase the risk for obesity and diabetes and increase the hence of having work-related or driving accidents, depression and personality disorders.

Sleep Apnea is a progressive condition and should be treated

\section{Diagnosis}

A sleep test, called polysomnography is usually done to diagnose sleep apnea. There are two kinds of polysomnograms. An overnight polysom-nography test involves monitoring 
brain waves, muscele tension, eye movemment, respiration, oxygen level in the blood and audio monitoring. (for snoring, gasping, etc.) The second kind of polysomnography test is a home monitoring tst. The electrodes are hooked to record the sleep with a computerized polysomnograph; so this can done while sleeping at home.

\section{Treatment}

While some of the people improve with lifestyle modification and use of simple mouthpieces, some may need, surgery, and/or breathing devices.

Mild Sleep Apnea is usually treated by some behavioral changes. Losing weight, sleeping on your side are often recommended. There are oral mouth devices (that help keep the airway open) on the market that may help to reduce snoring in three different ways. Some devices1 bring the jaw forward or 2 elevate the soft palate or 3 retain the tongue from falling back in the airway ad blocking breathing.

Moderate to severe Sleep Apnea is usually treated with a C-PAP (continous positive airway pressure). C-PAP is a machine that blows air into your nose via a nose mask, keeping the airway patent. For more severe apnea, there is a Bi-level (Bi-PAP) machine. The Bi-level machine is different in that it blows air at two different pressures during inspiration and expiration.

Fixing a deviated septum may help to open the nasal passages. Removing the tonsils and adenoids or polyps may help also. Children are much more likely to have their tonsils and adenoids removed.

\section{Tracheostomy}

During the day the valve is closed so the person can speak. At night, the valve is opened, thus avoiding the obstructions. This procedure is only used today as a last resort.

\section{Uvuloplatopharyngoplasty:}

The uvula is removed along with excess tissue in the pharynx. This surgery is usually done for patients who can't tolerate nasal CPAP. This surgery has mixed reviews, it helps in only $50 \%$ of the patients

There is also Laser Assisted Uvuloplasty to remove the palate with the help of laser and this relieves snoring successfully.

\section{Mandibularmyotomy:}

This procedure involves cutting a a rectangular piece of bone in the anterior portion of the mandible.

This pulls the tongue forward six to ten millimeters and almost always elominates the sleep-related obstruction. The procedure called radiofrequency volumetric tissue reduction of the palate uses radio waves to shrink tissue in air passages and eliminate snoring.

\section{Oral positive airway pressure:}

It is another new invention; its a dental appliance that conducts air through it. It may prove to be an alternative to the mask and headgear.

\section{CONCLUSION}

Sleep apnea is a serious condition which if untrated can lead to many cardiovascular complications.

One should get evaluated for sleep apnea from a sleep center if your snoring is lud enough to disturb your sleep or that of others, or if your experience shortness of breath that wakes you up, or you experience excessive daytime sleepiness, or your partner says that your breathing stops during sleep.

If you have high blood pressure and if you ever experience chest pain after waking up at night, you should get checked up for sleep apnea.

Initial approach should be standard conservative treatments like sleeping on your side, weight loss and avoiding alcohol before bed.

Consider continuous positive airway pressure (CPAP) if you have moderate sleep apnea. Surgical modalities are for severe cases not responding to these treatments.

\section{REFERENCE}

1. Wolk R, Gami As, Somers VK. Sleep Cardiovascular diseases. Currr Probl Cardiol 30.625, 2005,

2. gami As, Friedman PA, Chung MK et al. Therapy Insight Interaction between arterial fibrillation and obstructive sleep apnea. Nat Clin Pract Cardiovasc Med 2:145;2005.

3. Shamsuzzaman AS, Greb JB, Somes VK. Obstructive sleep apnea Implication for cardiac and vascular diseases. JAMA 290: 1906, 2003. 


\title{
Mitral valvotomy at Birendra Military Hospital
}

\author{
Rajbhandari GL* \\ *Birendra Military Hospital, nepal
}

\section{INTRODUCTION}

Shree Birendra Hospital is a 400 bedded referral military hosital situated in Kathmandu Nepal. It has been providing medical \& Surgical services to regular and retired army personnel and tieir families, for the last 75 years. It also provides specialized services in medical and surgical sub specialties like Neuro Surgery, Cardio-thoracic surgery, Uro-surgery. Gastro-enterology surgery, Laproscopic surgery, Cardiology, Gynaecology, Neuro-Psychiatry, Nephrology \& various other specialities. Diagnostic facilities are provided by advance Diagnostic facilities are provided by advance pathology and Radiology deparments (MRI CT scan, Bronchoscopy, G.I. Endoscopy, Echo-cardiocardiography etc).

Cardio-Thoracic Surgery unit started its services in this hospital from the year 2050 B.S. (1994 A.D) The first Cardiac surgery started with a successful operation for a patient with mitral stenosis by closed heart surgery (closed mitral valvotomy) in the year 2051 B.S, 23 Chaitra; (6th April, 1995 A.D.). cardiac problems are not usually encountered among regular army personnel due to strict medical screening during army recruitment so cardiac surgery is generally required for family members of the serving \& retired army personnel instead. 1

\section{MATERIALS AND METHODS}

From 2051 BS to 2057 BS (1995 to 2000 A.D.). 7 patients underwent Closed Mitral Volvotomy (CMV), among them 6 were female and 1 male. Their age ranged from 15 years to 40 years and there was no operative mortality. 2,3 The selection criteria for CMV was patient with tight mitral stenosis with Mitral Valve Area (MVA) less than or equal to $1 \mathrm{~cm} 2$ on Echocardiography, sinus rhythm, no clot in LA, ASO titre negative, no significant calcification of mitral Valve or mitral Regurgitation. Patients in clinical classification of NYHA class III to IV. 4,5

\section{RESULTS}

The routine surgical procedure was mitral volvotomy with digital commisurotomy followed by Tubbs dilator application up to $2.5 \mathrm{~cm}$. the result of CMV wassatisfactory clinically \& there were no significant post surgical mitral regurgitation. There was significant clinical improvement of patient post operatively \& patient were clinically in NYHA class I to II. There was no case of mitral re-stenosis after 4 years of CMV rest of 6 patients were clinically doing well during follow up of 5 years of operation. 6,7,8

On post operative Echocardiography MVA was found to be $1.8 \mathrm{~cm} 2$ to $2.2 \mathrm{~cm} 2$. one patient with post operative CMV developed mitral re-stenosis and had Percutaneous Transvenous Mitral Commissurotomy (PTMC) done at Shahid Gangalal Heart Centre in 2061.

\section{DISCUSSION}

History of mitral valvotomy starts back in $1923 \mathrm{AD}$ when Levin \& Cuttler successfully performed closed mitral valvotomy in an 11 year old girl with severe mitral stenosis. With the development of Heart Lung Machine Lillehei \& Kirklin performed open mitral valvotomy in 1955. since 1990 s with advancement of invasive cardiology successful percutaneous mitral commissurotomy was started in many centres of world. With the beginning of the Maoist insurgency from 2052 B.S. and with the involvement of nepal Army in insurgency control movment from 2058 B.S; this hospital was involved in management of insurgency casualties of Nepal army personnel \& Civillans. Thus the cardio thoracic surgical unit was involved in the management \& surgery of thoracic trauma patient during that period.

During last few years with the advancement of invasive cardiology \& development of non surgical Percutaneous Transvenous Mitral Commisurotomy (PTMC) in Nepal, most of the patients with mitral stenosis were referred to Shahid Gangalal Heart Centre. With the availability of non surgical PTMC, the oldest cardiac surgical procedure closed mitral valvotomy is almost obsolete in most of cardio-thoracic centres. Now closed mitral valvotomy is done only as an emergency surgical procedure in few cardio-thoracic centres in the world.

\section{REFERENCE}

1. Rajbhandary G.L. 1994. Mitral Valvotomy an Oldest Cardiac Surgical Procedure for Mitral Stenosis. MJSBH, Vold 2, No. 2 1999, Page no 22 to 28

2. Ellis LB etal 1973,15 to 20 years study of 1000 patients undergoing Mitral Valvotomy. Circulation, XL to 3724 consecutive patients Circulation 1983, 68 (5) 891

3. Goldman ME1986, Intra Operation Echocardiography for evaluation of Valvular regurgitation Experience in 263 patients Circulation 1986, 74, Supp. 1. 1-14

4. Anonymous 1973, Criteria Committee of New york Heart Association Nomenclauture criteria for diagnosis of disease of heart \& great Vessels. 7th Ed. Little Brown, Boston 1773, 286

5. Aventura AP, 1986, Closed Mitral Valvotomy, Rob \& Smiths Operative Surgery, Cardiac Surgery, Ed. Jamieson SW, Shumay NE, Butter Worth, London, 1986, 399.

6. Logan \& Turner 1959, surgical Treatment of Mitral Stenosis with particular reference to Mechanical Dilator. The lancet 1959,2 874

7. Rajbhandary G.L., Khan JS, Chema MA, 1994 early results of Closed Mitral Valvotomy \& open Mitral Valvotomy for Juvenile Mitral Stenosis in Pakistan. Proceedings of First international conference of Society of Surgenos of Nepal, Kathmandu, 1994

8. Loya YS 1991, Ballon Mitral Valvotomy, Pak J. Cardio, 1991, 41

9. Maskey A. 2005 Interventional Procedure at Sahid Gangalal National Heart Center 10th Anniversary Annual issue, 25 